\title{
EFEITOS DE PARÂMETROS DE APLICAÇÃO NA AÇÃO DESSECANTE DO HERBICIDA SULFOSATE SOBRE PLANTAS DE ARROZ ${ }^{1}$
}

\author{
NILSON G. FLECK ${ }^{2}$, LEANDRO VARGAS ${ }^{3}$, MARCOS M. DA CUNHA ${ }^{4}$, ANDRÉ ANDRES ${ }^{5}$
}

\section{RESUMO}

O controle de plantas daninhas do tipo gramíneas constitui-se em grande problema nos sistemas de cultivo mínimo, requerendo aplicações eficientes de herbicidas. Nesse sentido, parâmetros de tecnologia de aplicação como volume de calda, tamanho de gota e concentração herbicida exercem grande influência sobre a atividade desses produtos. O objetivo desse trabalho foi investigar os efeitos das interações da dose herbicida, sua concentração na solução e o volume do veículo diluente, sobre a eficiência do herbicida sulfosate, usando-se arroz como espécie reagente. $\mathrm{O}$ experimento foi conduzido durante a safra agrícola de 1994/95 na Estação Experimental do Arroz do IRGA, em Cachoeirinha, RS. Foram testados 18 tratamentos, constituídos por cinco doses de sulfosate $(1,7 ; 2,3 ; 3,0 ; 4,0$ e 4,7 1/ha de produto formulado); cinco volumes de diluente $(85 ; 115$; $150 ; 200$ e $2351 /$ ha) e cinco concentrações de produto na calda $(1,2 ; 1,5 ; 2,0 ; 2,6$ e $3,3 \% \mathrm{v} / \mathrm{v})$. Em cada condição um desses parâmetros era fixado e os outros dois variavam. Ao se manter constante o volume de calda, não houve diferenças, na última avaliação, entre as doses 3,0 ; 4,0 e 4,7 1/ha de sulfosate, quando todas alcançaram nível ao redor de $90 \%$ de controle do arroz. Já quando se manteve constante a concentração herbicida, as respostas às doses foram não significativas. Apenas na última avaliação observou-se desempenho inferior da menor dose. Na terceira condição, quando se manteve constante a dose herbicida, observou-se que o sulfosate foi mais atuante quando aplicado nas maiores concentrações. Foram alcançados níveis de controle ao redor de $90 \%$ com doses de sulfosate de 3 1/ha ou superiores, ou com concentrações herbicidas de $2 \%$ ou maiores. Isso demonstra que parâmetros de tecnologia de aplicação de sulfosate podem ser alterados no sentido de se obter maior eficiência na sua ação.

Palavras chave: Dose herbicida, concentração na calda, volume de calda, tamanho da gota.

\section{ABSTRACT \\ Effects of application parameters on desiccation action of herbicide sulfosate on rice}

Control of weed grass species constitutes a great problem in reduced tillage systems, requiring efficient herbicide application. In this sense, application parameters like spray volume, dropplet size, and herbicide concentration may exert great influence on activity of these products. The objective of this research was to investigate the interaction effects

\footnotetext{
${ }^{1}$ Recebido para publicação em 12/11/96 e na forma revisada em 02/09/99.

2 Eng. Agr., Ph. D., Prof. Aposentado do Depto. de Plantas de Lavoura, Fac. de Agronomia da UFRGS. Bolsista do CNPq. Cx. Postal 776, CEP 95501-970, Porto Alegre, RS.

${ }^{3}$ Eng. Agr., M. Sc., Bolsista Recém-Mestre da FAPERGS no Depto. de Plantas de Lavoura da Fac. de Agronomia da UFRGS, Porto Alegre, RS.

${ }^{4}$ Eng. Agr., Aluno do Programa de Pós-Graduação em Agronomia, Fac. de Agronomia da UFRGS, Porto Alegre, RS.

${ }^{5}$ Eng. Agr., M. Sc., Pesquisador do Instituto Riograndense do Arroz, Cachoeirinha, RS.
} 
of herbicide rate, herbicide concentration, and diluent volume, on sulfosate efficiency, using rice as test plant. The experiment was conducted during the 1994/95 growing season at the IRGA Rice Experimental Station, in Cachoeirinha, RS, Brazil. Eighteen treatments were tested, performed by five sulfosate rates $(1.7 ; 2.3 ; 3.0 ; 4.0$; and 4.7 $1 /$ ha of formulated product), five diluent volumes $(85 ; 115 ; 150 ; 200 ;$ and $235 \mathrm{l} / \mathrm{ha})$, and five product concentrations in spray solution $(1.2 ; 1.5 ; 2.0 ; 2.6$; and $3.3 \% \mathrm{v} / \mathrm{v})$. In each condition, one of these parameters was fixed, whereas the other two varied. When maintained spray volume constant, there was no difference, at the last evaluation, among sulfosate rates of $3.0 ; 4.0$; and $4.7 \mathrm{l} / \mathrm{ha}$,

\section{INTRODUÇÃO}

O controle de plantas daninhas do tipo gramíneas e de plantas cultivadas de ocorrência espontânea nas culturas de cereais tem-se constituído em grande problema no sistema de cultivo mínimo. O sistema de produção das culturas através do método de semeadura direta ou de preparo mínimo do solo, denominados sistemas conservacionistas, estão sendo utilizados cada vez mais na produção de arroz irrigado no estado do Rio Grande do Sul, visando especialmente manejar as infestações de arroz daninho (vermelho, preto ou branco espontâneo) nessas áreas. Contudo, mesmo com a adoção das técnicas de semeadura direta ou de cultivo mínimo, o manejo destas plantas daninhas ainda necessita de medidas de controle químico. Esses sistemas de produção requerem aplicações cada vez mais eficientes de herbicidas de pós-emergência, situações em que as características de aplicação, como volume de calda, tamanho de gota e concentração herbicida nas gotas, parecem exercer grande influência sobre a atividade dos produtos.

Dentre os herbicidas utilizados com tal finalidade, destacam-se dois compostos derivados da glicina, glyphosate e sulfosate, que são absorvidos pela folhagem, apresentam ação sistêmica e ausência de seletividade para a maioria das espécies, constituindo-se em alternativas when all these reached rice control around 90\%. When herbicide concentration was maintained constant, the responses to herbicide rates were not significant. In the third condition, when herbicide rate was fixed, it was observed that sulfosate action increased at higher concentrations. Control levels around $90 \%$ were reached with herbicide concentration of $2 \%$ or higher. This demonstrates that application parameters of sulfosate may be modified in order to obtain greater efficiency in its action.

Key words: Herbicide rate, spray herbicide concentration, spray volume, dropplet size.

eficientes para controle de espécies anuais, bienais e perenes. Foi demonstrado que a fitotoxicidade do glyphosate e do sulfosate foram similares em trigo quando utilizados em iguais doses, volumes de veículo, qualidade da água e concentrações de ácido sulfúrico (Carlson \& Burnside, 1984).

Diversos herbicidas têm mostrado variação no controle de espécies daninhas em função do volume de diluente, da concentração do herbicida e do tamanho da gotícula herbicida que é depositada sobre a folhagem da planta (Jordan, 1981). Modificações na aplicação de herbicidas sistêmicos não-seletivos, como redução do volume do veículo de aspersão, têm permitido reduções na dose herbicida, resultando em redução de custo, enquanto mantém nível adequado de controle de plantas daninhas (Carlson \& Burnside, 1984). A importância da utilização de baixos volumes nas aplicações está diretamente relacionado com o maior rendimento dos pulverizadores, sendo possível aplicar uma maior área por tanque (Marochi, 1996).

Ensaios de campo e de casa-de-vegetação têm mostrado que aplicações de glyphosate em baixos volumes, menores que 100 1/ha, exibem maior fitotoxicidade do que altos volumes de diluente (Sandberg et al., 1978; Ambach \& Ashford, 1982; Buhler \& Burnside, 1987). 
Diversas pesquisas têm demonstrado aumentos significativos na fitotoxicidade de herbicidas como glyphosate e sulfosate pela redução do volume do veículo de aspersão (Ambach \& Ashford, 1982; Jordan, 1981; Bryson et al., 1994). Redução de volume de calda de 190 para 96 1/ha resultou em aumento de fitotoxicidade para ambos os herbicidas quando usados a $100 \mathrm{~g} / \mathrm{ha}$ (Carlson \& Burnside, 1984).

O volume aplicado pode ser reduzido, obtendo-se uma perfeita cobertura da folhagem através da adequação do tamanho das gotas (McWhorter \& Gebhardt, 1987). O tamanho de gota e o seu volume estão matematicamente relacionados, de modo que o número de gotas que pode ser produzido a partir de um determinado volume de líquido varia inversamente com o cubo do diâmetro da gota (McWhorter \& Gebhardt, 1987). Com a redução do volume de aplicação, ocorre aumento da concentração de adjuvante e de ingrediente ativo do herbicida na gota de aspersão. Esta mudança na composição da gota pode influir o suficiente para alterar a absorção e a translocação do herbicida na planta, de modo a afetar a sua eficiência (Ambach \& Ashford, 1982).

O tamanho da gota afeta a absorção e a deposição das gotas sobre a folhagem das plantas (McWhorter \& Gebhardt, 1987). Gotas pequenas são retidas com maior facilidade sobre a superfície das folhas, de modo que os herbicidas podem penetrar e translocar-se mais rapidamente do que se contidos em gotas grandes (Prasad \& Cadogan, 1992). Gotas pequenas também são mais fitotóxicas devido a maior cobertura da folhagem, o que proporciona maior absorção e translocação do herbicida (Prasad \& Cadogan, 1992; Al-Khatib et al., 1994). A absorção de thifensulfuron e de glyphosate aumentaram com uso de gotas pequenas e concentradas (Prasad \& Cadogan, 1992; Al-Khatib et al., 1994).

Altas concentrações de herbicida na calda podem superar o efeito do tamanho da gota (Prasad \& Cadogan, 1992). Porém, gotas pequenas e concentradas causam maior dano aos tecidos vegetais do que gotas maiores e menos concentradas (Al-Khatib et al., 1994). Concentrações elevadas de glyphosate na gota podem causar danos nos tecidos vegetais, reduzindo a absorção e a translocação (Leif III \&
Oelke, 1990), o que demonstra que altas concentrações podem ser negativas à atividade do herbicida. Decidir qual o tamanho ótimo das gotas para máxima eficiência e tolerância da cultura é difícil, mas esse fator deve ser levado em consideração, já que se relaciona com o custo de aplicação (Prasad \& Cadogan, 1992).

O tipo de bico utilizado na aplicação definirá o tamanho de gotas formadas. O tamanho de gotas produzido por um bico necessita ser conhecido para tornar possível ajustar o tipo de bico que proporcione o tamanho ideal de gota para se obter a cobertura desejada (Mcwhorter \& Gebhardt, 1987). Ao pesquisar o controle de arroz vermelho com sulfosate e glyphosate, Foloni (1996) obteve maior eficiência no controle dessa espécie quando esses herbicidas foram aplicados com bicos DG (anti-deriva) das séries $110.02 \mathrm{e}$ 110.03 , com volumes de calda de 150 e $287 \mathrm{l} / \mathrm{ha}$, respectivamente.

Embora, em geral, haja concordância de que a dose herbicida mostre maior atividade quando o volume do diluente é reduzido, ainda há alguma divergência em relação ao papel desempenhado pelo tamanho, densidade e composição das gotículas de aspersão sobre a eficiência dos herbicidas e mesmo quanto à tolerância das culturas. $\mathrm{O}$ objetivo desse trabalho foi investigar o efeito das interações da dose herbicida, sua concentração na solução e o volume do veículo diluente, sobre a eficiência do herbicida sulfosate, usando-se arroz daninho como espécie reagente.

\section{MATERIAL E MÉTODOS}

$\mathrm{O}$ experimento foi conduzido no ano agrícola de 1994/95, na Estação Experimental do Instituto Riograndense do Arroz (IRGA), localizada no município de Cachoeirinha, RS, em solo da unidade de mapeamento Vacacaí. Para comparar os tratamentos foi utilizado delineamento de blocos ao acaso, com quatro repetições. As parcelas apresentaram área útil de $18 \mathrm{~m}^{2}(3 \times 6 \mathrm{~m})$.

Foram testados 18 tratamentos (Tabela 1), sendo cinco doses de sulfosate $(1,7 ; 2,3 ; 3,0 ; 4,0$ e 4,7 1/ha do produto comercial); cinco volumes de 
calda $(85,115,150,200$ e $2351 /$ ha); e cinco concentrações de produto na calda $(1,2 ; 1,5 ; 2,0$; 2,6 e $3,3 \% \mathrm{v} / \mathrm{v}$ ), calculadas pela divisão da dose do produto comercial pelo volume de diluente. Em cada situação, um desses parâmetros foi fixado e os outros variavam. Acrescentaram-se três padrões para comparação: glyphosate a 3 l/ha $(2 \% \mathrm{v} / \mathrm{v})$; paraquat a $21 / \mathrm{ha}(1 \% \mathrm{v} / \mathrm{v})$ e testemunha sem herbicida. Os tratamentos herbicidas foram aplicados sobre plantas de arroz cultivado (cultivar BR-IRGA 410), simulando-se uma infestação de arroz vermelho/preto.

A semeadura do arroz foi realizada no dia 29/11/94, em linhas afastadas de $15,8 \mathrm{~cm}$ entre si. A emergência das plantas ocorreu em 09/12/94. A adubação química foi realizada na instalação do experimento e se constituiu na distribuição de 50 e $90 \mathrm{~kg} / \mathrm{ha}$ de superfosfato triplo e de cloreto de potássio, respectivamente.

Tabela 1. Tratamentos testados na avaliação de parâmetros de tecnologia de aplicação na ação dessecante do herbicida sulfosate sobre arroz (Orysa sativa )' IRGA, Cachoeirinha/RS, $1994 / 95$.

\begin{tabular}{|c|c|c|c|c|}
\hline \multicolumn{2}{|c|}{ Dose de produto } & \multirow{2}{*}{$\begin{array}{l}\text { Concentração de } \\
\text { produto na calda } \\
(\%-\mathrm{v} / \mathrm{v})\end{array}$} & \multirow{2}{*}{$\begin{array}{c}\text { Volume de calda } \\
\text { por área } \\
\text { (1/ha) } \\
\end{array}$} & \multirow{2}{*}{$\begin{array}{c}\text { Bicos de aspersão } \\
\text { tipo leque usados } \\
\text { na aplicação }\end{array}$} \\
\hline Comercial (ml/ha) & Ativo (g/ha) & & & \\
\hline \multicolumn{5}{|c|}{ Mantendo constante o volume de calda em 150 1/ha } \\
\hline 4700 & 1550 & 3,13 & 150 & 110.02 \\
\hline 4000 & 1320 & 2,67 & 150 & 110.02 \\
\hline 3000 & 990 & 2,00 & 150 & 110.02 \\
\hline 2300 & 760 & 1,53 & 150 & 110.02 \\
\hline 1700 & 560 & 1,13 & 150 & 110.02 \\
\hline \multicolumn{5}{|c|}{ Mantendo constante a concentração na calda em $2 \% \mathrm{v} / \mathrm{v}$} \\
\hline 4700 & 1550 & 2,00 & 235 & 110.04 \\
\hline 4000 & 1320 & 2,00 & 200 & 110.03 \\
\hline 3000 & 990 & 2,00 & 150 & 110.02 \\
\hline 2300 & 760 & 2,00 & 115 & 110.015 \\
\hline 1700 & 560 & 2,00 & 85 & 110.01 \\
\hline \multicolumn{5}{|c|}{ Mantendo constante a dose herbicida em 3,0 1/ha } \\
\hline 3000 & 990 & 3,53 & 85 & 110.01 \\
\hline 3000 & 990 & 2,61 & 115 & 110.015 \\
\hline 3000 & 990 & 2,00 & 150 & 110.02 \\
\hline 3000 & 990 & 1,50 & 200 & 110.03 \\
\hline 3000 & 990 & 1,28 & 235 & 110.04 \\
\hline \multicolumn{5}{|c|}{ Padrões de comparação } \\
\hline \multicolumn{5}{|l|}{ glyphosate } \\
\hline 3000 & 1080 & 2,00 & 150 & 110.02 \\
\hline \multicolumn{5}{|c|}{ paraquat + espalhante-adesivo a $0,1 \%$} \\
\hline 2000 & 400 & 1,00 & 200 & 110.03 \\
\hline
\end{tabular}

${ }^{1}$ Tratamentos aplicados sobre plantas de arroz quando se encontravam no estádio de dois afilhos. 
As aplicações herbicidas ocorreram no dia 28/12/1994, ocasião em que as plantas de arroz encontravam-se no estádio de dois afilhos. Para tal, foi utilizado aspersor costal de precisão, pressurizado com $\mathrm{CO}_{2}$, mantendo-se pressão constante de $160 \mathrm{kPa}$ durante as aplicações. $\mathrm{O}$ aspersor estava munido de bicos TeeJet, formadores de jato leque das séries 110.01; $110.015 ; \quad 110.02 ; \quad 110.03$ e 110.04 , que proporcionaram, respectivamente, os volumes de aspersão de $85 ; 115 ; 150 ; 200$ e 235 1/ha. Esses tratamentos foram aplicados no horário entre 8:15 e 9:45 horas. Ocorreu chuva a partir de 10 a 12 horas após as aplicações.

Os efeitos (fitotoxicidade) dos tratamentos foram avaliados visualmente, em cinco épocas, aos $5,10,15,20$ e 25 dias após aplicação dos tratamentos (DAT), de modo comparativo à testemunha não-tratada, utilizando-se escala percentual, onde nota zero significou nenhum efeito de dano ao arroz e nota 100 representou a morte das plantas. Cada avaliação teve a participação de dois ou de três técnicos, que realizaram avaliações independentes, cujos valores foram somados e procedidas as médias para cada repetição. Os valores percentuais obtidos foram transformados pela raiz quadrada antes da realização da análise de variância. Quando constatada significância estatística, efetuou-se a complementação da análise através da comparação das médias dos tratamentos pelo teste de Duncan, ao nível de 5\% de probabilidade. Além disso, procedeu-se análise de regressão polinomial, utilizando-se os modelos linear e quadrático, com e sem transformação das variáveis dependente e independente através de logaritmo e raiz quadrada.

\section{RESULTADOS E DISCUSSÃO}

Os resultados mostraram rápido efeito herbicida do paraquat, produto com ação de contato, e lenta atividade dos produtos sistêmicos (glyphosate e sulfosate) (Tabela 2). Paraquat causou níveis de dano moderado às plantas de arroz $(69,64$ e $59 \%)$ nas três primeiras avaliações
(5, 10 e 15 DAT), e aumentando consideravelmente aos 20 e 25 DAT (91 e 96\%). No entanto, nenhum dos demais tratamentos atingiu nível equivalente ao de paraquat nas duas primeiras avaliações. Em comparação ao tratamento padrão glyphosate, em todas as avaliações ocorreu ação similar desse produto com a de sulfosate, quando ambos foram usados sob as mesmas condições de aplicação.

Ao se manter constante a variável volume de calda em 150 1/ha, constatou-se resposta do arroz à dose de sulfosate. A menor dose desse herbicida (1,7 1/ha) diferiu da maior dose já na primeira avaliação, foi inferior às duas maiores doses na segunda avaliação e ampliou sua condição de inferioridade a todas as demais doses testadas nas três últimas avaliações. Não houve diferença significativa entre doses de 3,0; 4,0 e 4,7 1/ha de sulfosate nas três últimas avaliações (15, 20 e 25 DAT), período em que todas alcançaram nível elevado de dano à espécie reagente. Já a dose de 2,3 1/ha de sulfosate diferiu da maior dose apenas nas duas últimas avaliações. Essa dose aplicada com 150 l/ha de calda apresentou controle satisfatório de arroz, próximo a 90\%, somente quando a concentração do herbicida na calda esteve em $2 \%$ ou acima, indicando efeito positivo da maior concentração sobre o controle do arroz.

Já quando se manteve constante a variável concentração herbicida na calda em $2 \%$, as respostas à variação de dose do produto foram menores. De um modo geral, não ocorreram diferenças entre doses de sulfosate durante as quatro primeiras avaliações (até $20 \mathrm{DAT}$ ). Apenas na última avaliação, aos 25 DAT, observou-se que a dose menor (1,7 1/ha) mostrou desempenho inferior às três doses maiores, confirmando assim os resultados relatados anteriormente. $\mathrm{O}$ aumento na concentração herbicida proporcionou aumento na fitotoxicidade, principalmente para a menor dose de sulfosate (1,7 1/ha), a qual não diferiu, aos 25 DAT, do tratamento padrão de glyphosate (3 1/ha), fato constatado somente quando a concentração foi mantida constante em $2 \%$. 
É interessante ressaltar que, ao se comparar a dose menor (1,7 1/ha) nas duas situações colocadas acima, verificou-se para as duas últimas avaliações, efeito positivo do aumento da concentração de sulfosate na calda, pois o efeito do produto aplicado à $2 \%$ foi superior à sua ação na concentração de apenas 1,1\%. Comportamento similar também foi constatado por Prasad \& Cadogan, (1992) e por Bryson et al., (1994).

Tabela 2. Efeitos de parâmetros de tecnologia de aplicação na ação dessecante do herbicida sulfosate sobre arroz (Oryza sativa), IRGA, Cachoeirinha/RS, 1994/95.

\begin{tabular}{|c|c|c|c|c|c|c|c|c|}
\hline \multicolumn{2}{|c|}{$\begin{array}{l}\text { Doses de } \\
\text { produto }\end{array}$} & \multirow{2}{*}{$\begin{array}{l}\text { Concen- } \\
\text { tração de } \\
\text { produto } \\
\text { na calda } \\
(\%-\mathrm{v} / \mathrm{v})\end{array}$} & \multirow{2}{*}{$\begin{array}{l}\text { Calda por } \\
\text { área } \\
\text { (1/ha) }\end{array}$} & \multicolumn{5}{|c|}{$\begin{array}{l}\text { Avaliações de } \\
\text { controle }^{1}\end{array}$} \\
\hline $\begin{array}{c}\text { Comercial } \\
(\mathrm{ml} / \mathrm{ha})\end{array}$ & $\begin{array}{l}\text { Ativo } \\
(\mathrm{g} / \mathrm{ha}) \\
\end{array}$ & & & $05^{2}$ & 10 & 15 & 20 & 25 \\
\hline \multicolumn{9}{|c|}{ Mantendo constante o volume de calda em 150 1/ha } \\
\hline 4700 & 1550 & 3,13 & 150 & $29 b^{3}$ & $48 \mathrm{~b}$ & $72 \mathrm{a}$ & $84 \mathrm{bc}$ & $94 \mathrm{ab}$ \\
\hline 4000 & 1320 & 2,67 & 150 & $26 \mathrm{bc}$ & $41 \mathrm{bcd}$ & $66 \mathrm{ab}$ & $83 \mathrm{bcd}$ & $89 \mathrm{abc}$ \\
\hline 3000 & 990 & 2,00 & 150 & $27 \mathrm{~b}$ & 35 cdef & $65 a b c$ & $82 \mathrm{bcd}$ & $88 \mathrm{abc}$ \\
\hline 2300 & 760 & 1,53 & 150 & $25 \mathrm{bcd}$ & 33 def & $65 \mathrm{abc}$ & $77 \mathrm{de}$ & $81 \mathrm{~cd}$ \\
\hline 1700 & 560 & 1,13 & 150 & $21 \mathrm{~cd}$ & $31 \mathrm{f}$ & $52 \mathrm{~d}$ & $69 \mathrm{f}$ & $65 \mathrm{e}$ \\
\hline \multicolumn{9}{|c|}{ Mantendo constante a concentração na calda em $2 \%$ v/v } \\
\hline 4700 & 1550 & 2,00 & 235 & $28 \mathrm{~b}$ & 39 cde & $68 \mathrm{ab}$ & $82 \mathrm{bcd}$ & $88 \mathrm{abc}$ \\
\hline 4000 & 1320 & 2,00 & 200 & $27 \mathrm{~b}$ & 40 bcde & $72 \mathrm{a}$ & $85 \mathrm{~b}$ & $92 \mathrm{ab}$ \\
\hline 3000 & 990 & 2,00 & 150 & $27 \mathrm{~b}$ & $35 \mathrm{cdef}$ & $65 \mathrm{abc}$ & $82 \mathrm{bcd}$ & $88 \mathrm{abc}$ \\
\hline 2300 & 760 & 2,00 & 115 & $25 \mathrm{bcd}$ & 38 cdef & $64 a b c$ & $81 \mathrm{bcd}$ & 85 bcd \\
\hline 1700 & 560 & 2,00 & 85 & $24 \mathrm{bcd}$ & 35 cdef & $59 \mathrm{bcd}$ & $80 \mathrm{bcd}$ & $79 \mathrm{~d}$ \\
\hline \multicolumn{9}{|c|}{ Mantendo constante a dose herbicida em 3,0 1/ha } \\
\hline 3000 & 990 & 3,53 & 85 & $27 \mathrm{~b}$ & $43 \mathrm{bc}$ & $71 \mathrm{a}$ & $84 \mathrm{bc}$ & $93 \mathrm{ab}$ \\
\hline 3000 & 990 & 2,61 & 115 & $28 \mathrm{~b}$ & 35 cdef & $69 \mathrm{ab}$ & $81 \mathrm{bcd}$ & $92 \mathrm{ab}$ \\
\hline 3000 & 990 & 2,00 & 150 & $27 \mathrm{~b}$ & $35 \mathrm{cdef}$ & $65 \mathrm{abc}$ & $82 \mathrm{bcd}$ & $88 \mathrm{abc}$ \\
\hline 3000 & 990 & 1,50 & 200 & $26 \mathrm{bc}$ & 32 ef & $61 \mathrm{abcd}$ & 78 cde & $81 \mathrm{~cd}$ \\
\hline 3000 & 990 & 1,28 & 235 & $21 \mathrm{~cd}$ & 30 ef & $54 \mathrm{~cd}$ & 73 ef & $70 \mathrm{e}$ \\
\hline \multicolumn{9}{|c|}{ Padrões de comparação } \\
\hline \multicolumn{9}{|l|}{ Glyphosate } \\
\hline 3000 & 1080 & 2,00 & 150 & $24 \mathrm{bcd}$ & 39 cde & $64 a b c$ & $82 \mathrm{bcd}$ & $86 \mathrm{bcd}$ \\
\hline \multicolumn{9}{|c|}{ Paraquat + espalhante-adesivo a $0,1 \%$} \\
\hline 2000 & 400 & 1,00 & 200 & $69 \mathrm{a}$ & $64 \mathrm{a}$ & $59 \mathrm{bcd}$ & $91 \mathrm{a}$ & $96 \mathrm{a}$ \\
\hline \multicolumn{9}{|c|}{ Testemunha sem uso de herbicida } \\
\hline Coeficiente & le variaçã & & & 5,6 & 6,4 & 5,5 & 4,6 & 5,8 \\
\hline
\end{tabular}


$\mathrm{Na}$ terceira condição, quando se manteve constante a dose herbicida em 3 1/ha, de um modo geral, verificou-se que o sulfosate aplicado com menor volume de aspersão, conseqüentemente em maiores concentrações na calda, foi mais atuante em sua ação. Especialmente a menor concentração $(1,3 \%)$ mostrou ação herbicida inferior ao produto aplicado em concentração de $2 \%$ ou acima, as quais mostraram os maiores níveis de controle. Da mesma forma, as duas últimas avaliações também indicaram que o desempenho de glyphosate a $2 \%$ foi superior ao de sulfosate a 1,3\% quando ambos foram usados na mesma dose ( 3 1/ha). Esse fato evidencia novamente aumento da fitotoxicidade herbicida com o aumento da sua concentração na calda devido à redução do volume do diluente, o que concorda com os resultados relatados por Buhler \& Burnside (1987). Alta concentração herbicida na solução pode aumentar a fitotoxicidade devido a maior absorção de herbicida por unidade de volume da solução que penetra na folha, além de criar um gradiente de concentração entre a solução e a folha, o que aumenta a taxa de difusão do herbicida para o interior da folha (Buhler \& Burnside, 1984).

Além da maior concentração herbicida na calda, o tipo de bico usado também influi na ação dos herbicidas, conforme foi relatado por Foloni (1996). Nesse trabalho foi observado maior efeito fitotóxico do sulfosate, na concentração constante de $2 \%$ do produto na calda, quando foram utilizados bicos da série 110.01; 110.015 e 110.02, que propiciaram volumes de aspersão de $85,115 \mathrm{e}$ 150 1/ha, respectivamente, em relação aos bicos 110.03 e 110.04, os quais proporcionaram volumes de aspersão de 200 e 235 1/ha. Esse efeito, tanto pode ser devido aos diferentes volumes aspergidos pelos diferentes tipos de bico como pelos diferentes tamanhos de gotas formadas, ou ainda devido a ambos. Inerente ao volume de calda aspergida, desponta o fator concentração do herbicida nas gotas. Os bicos $110.01 ; 110.015$ e 110.02 , ao serem operados à pressão de $160 \mathrm{kPa}$, produzem maior quantidade de gotas com tamanho médio. Já os bicos 110.03 e 110.04 , com utilização da mesma pressão, produzem maior quantidade de gotas de tamanho grande (Spraying Systems, 1994). Bicos com orifício pequeno produzem grande quantidade de gotas pequenas (Buhler \& Burnside, 1983), assim como quantidades iguais de herbicida são aplicadas com gotas pequenas e concentradas, resultam em maior fitotoxicidade do que gotas grandes e menos concentradas (Buhler $\&$ Burnside, 1984).

O tamanho das gotas é dependente do volume de aspersão, quando se mantém o número de gotas constante por área; dessa forma, maiores volumes proporcionam aspersões com gotas grandes, enquanto volumes menores dão origem a aspersões que fornecem gotas pequenas. Com isso, os bicos que proporcionaram os menores volumes de calda $(85,115$ e 150 l/ha) produziram gotas médias, enquanto os bicos que forneceram volumes de aspersão de 200 e 235 1/ha produziram gotas maiores.

As gotas de tamanho médio podem apresentar algumas vantagens sobre gotas pequenas ou grandes. As gotas pequenas podem evaporar com facilidade, não atingindo o alvo (Prasad \& Cadogan, 1992), ou causar danos aos tecidos vegetais, reduzindo a absorção e a translocação do produto, devido à alta concentração herbicida que contém (Al-Khatib et al., 1994). Embora, gotas pequenas proporcionam maior cobertura foliar, o que aumenta a absorção e a translocação do herbicida (Buhler \& Burnside, 1984; Prasad \& Cadogan, 1992). Por outro lado, gotas grandes proporcionam menor cobertura e, com isso, menor absorção e translocação do produto (Prasad \& Cadogan, 1992; Al-Khatib et al., 1994), além de ocorrer maior probabilidade de escorrimento desse tipo de gota da superfície da folha. $\mathrm{O}$ escorrimento se torna significativo quando o volume aspergido supera 190 1/ha (Sandberg et al. 1978). Portanto, o tamanho ideal de gotas é aquele que proporcione a melhor cobertura foliar possível e contenha concentração que favoreça a absorção; nesse caso, seriam preferidas gotas pequenas e médias. 
O número de gotas é outro fator influenciado pelo tamanho das gotas formadas. Bicos que formam gotas grandes produzem menor número de gotas, propiciando cobertura foliar insatisfatória, em comparação aos bicos que produzem gotas menores, os quais proporcionam melhor cobertura foliar. Gotas pequenas aumentam a cobertura foliar, o que resulta em maior absorção por unidade de tempo devido ao aumento na superfície de contato (Buhler \& Burnside, 1984). Deste modo, a maior fitotoxicidade observada com bicos de menor vazão pode ser devida ao tamanho de gotas formadas, o que propiciou melhor cobertura foliar, e ao volume de aspersão; nesse caso, basicamente devido à maior concentração do herbicida na calda e na gota. Isso demonstra que a maior atividade do herbicida sulfosate foi atingida com aplicações que utilizaram bicos $110.01 ; 110.015$ e 110.02 , os quais formaram os menores volumes de aspersão, possibilitando assim aumento da concentração do herbicida na calda.

Com relação à análise de regressão, a Figura 1 contém as curvas de regressão estimadas para as várias situações estudadas. Os coeficientes de determinação da regressão para os modelos que melhor estimaram os pontos foram relativamente baixos (Tabela 3). Apenas em três situações, todas verificadas aos $25 \mathrm{DAT}$, obtiveram-se coeficientes na faixa entre 70 e $80 \%$; para todos os demais casos eles foram inferiores a 70\%. Nessa Figura, observa-se que nas primeiras avaliações, quando houve regressão significativas entre variáveis, as relações apresentaram-se lineares. E ainda, que ao menos na última avaliação, essas regressões assumiram relação quadrática.

A relação linear inicialmente observada (até os 15 ou 20 DAT) mostra que o incremento da variável em questão trouxe um aumento correspondente na ação do herbicida. Já o relacionamento quadrático observado aos 20 ou 25 DAT, indica que a partir de determinado valor da variável o efeito se estabilizou, não determinando ganho adicional com incremento da mesma. Podese visualizar nessas curvas que aos $25 \mathrm{DAT}$, ao se considerar como aceitável um nível de controle de 90\%, para dose herbicida esse ponto situa-se entre 3,0 e 3,5 1/ha, e que para concentração herbicida ele está situado entre 2,25 e 2,5\%. Em outras palavras, não há ganho adicional em se utilizar dose de sulfosate superior a 3,5 1/ha ou concentração herbicida na calda acima de $2,5 \%$ para se atingir grau de controle de arroz de $90 \%$.

Nota-se na Figura 1 que ocorreu acréscimo do controle de arroz de uma avaliação para a seguinte; no entanto, constata-se que, para as duas últimas avaliações, as duas doses e as duas concentrações menores já haviam atingido seu efeito máximo aos 20 DAT, não ocorrendo mais nenhum incremento posterior. Já as doses de 3,0 1/ha ou superiores ou concentrações de $2 \%$ ou maiores ainda experimentaram incremento na atividade herbicida nesse intervalo final de 5 dias.

Observa-se também que, ao se manter a concentração herbicida constante na calda em $2 \%$ (Figura 1a), para a maioria das avaliações não se constatou significância para os modelos de regressão testados quanto à variação da dose herbicida. Isso indica que a esta concentração, geralmente há pouca expectativa de variação do efeito com aumento da dose herbicida.

Neste trabalho foram alcançados níveis de controle de arroz ao redor de $90 \%$ com doses de sulfosate de 3,0 1/ha ou superiores, ou com concentração herbicida de $2 \%$ ou maiores. Dose de $2,31 /$ ha ou concentração de $1,5 \%$ podem alcançar nível de controle de aproximadamente $80 \%$. Já a dose de sulfosate de $1,7 \mathrm{l} / \mathrm{ha}$ ou concentração na calda inferior a 1,5\% mostraramse insuficientes no seu efeito herbicida pleno sobre arroz. Esses resultados permitem concluir que parâmetros de aplicação do herbicida sulfosate podem ser alterados no sentido de se obter maior eficiência em sua ação. Isso pode ser conseguido através da redução do volume de calda aplicada, o que resulta em aumento da concentração do produto na solução de aspersão. O aumento da concentração de produto na calda proporciona maior eficiência herbicida ao sulfosate, representando maior economia, tanto do produto quanto de água para aspersão e, conseqüentemente, pode permitir até certa redução da dose herbicida. 
Tabela 3. Equações de regressão e coeficientes de determinação obtidos para parâmetros de tecnologia de aplicação avaliados na ação dessecante do herbicida sulfosate sobre arroz (Oryza sativa), IRGA, Cachoeirinha/RS, 1994/95.

\begin{tabular}{|c|c|c|}
\hline Avaliaçõos ${ }^{(1)}$ & Equações de regressão & Valores de $\mathrm{r}^{2}$ \\
\hline \multicolumn{3}{|c|}{ Mantendo constante o volume de calda em 150 1/ha } \\
\hline $5 \mathrm{DAT}$ & $\mathrm{Y}=19,17+0,002 \operatorname{dose}^{(2)}$ & 0,17 \\
\hline $10 \mathrm{DAT}$ & $\mathrm{Y}=20,80+0,005$ dose & 0,56 \\
\hline $15 \mathrm{DAT}$ & $\mathrm{Y}=50,14+0,005$ dose & 0,31 \\
\hline 20 DAT & $\mathrm{Y}=40,95+0,022$ dose $-0,000003$ dose $^{2}$ & 0,61 \\
\hline $25 \mathrm{DAT}$ & $\mathrm{Y}=21,54+0,034$ dose $-0,000004$ dose $^{2}$ & 0,71 \\
\hline $5 \mathrm{DAT}$ & $\mathrm{Y}=19,18+3,11$ concentração $^{(3)}$ & 0,17 \\
\hline $10 \mathrm{DAT}$ & $Y=20,85+8,15$ concentração & 0,56 \\
\hline $15 \mathrm{DAT}$ & $Y=50,17+6,77$ concentração & 0,30 \\
\hline $20 \mathrm{DAT}$ & $Y=41,10+32,13$ concentração $-5,94$ concentração ${ }^{2}$ & 0,61 \\
\hline 25 DAT & $Y=21,72+50,34$ concentração $-8,86$ concentração ${ }^{2}$ & 0,71 \\
\hline \multicolumn{3}{|c|}{ Mantendo constante a concentração herbicida na calda em $2 \%$ v/v } \\
\hline $5 \mathrm{DAT}$ & $\mathrm{Y}=26,98($ dose $)$ & $\mathrm{NS}^{(4)}$ \\
\hline 10 DAT & $\mathrm{Y}=37,88($ dose $)$ & NS \\
\hline 15 DAT & $Y=54,91+0,003$ dose & 0,25 \\
\hline 20 DAT & $\mathrm{Y}=81,95($ dose $)$ & NS \\
\hline 25 DAT & $\mathrm{Y}=50,22+0,0217$ dose $-0,000003$ dose $^{2}$ & 0,45 \\
\hline \multicolumn{3}{|c|}{ Mantendo constante a dose herbicida em 3 1/ha } \\
\hline $5 \mathrm{DAT}$ & $Y=27,04$ (concentração) & NS \\
\hline 10 DAT & $Y=23,83+5,25$ concentração & 0,45 \\
\hline $15 \mathrm{DAT}$ & $Y=49,24+6,85$ concentração & 0,36 \\
\hline $20 \mathrm{DAT}$ & $Y=71,22+3,94$ concentração & 0,37 \\
\hline 25 DAT & $Y=26,60+45,54$ concentração $-7,59$ concentração ${ }^{2}$ & 0,78 \\
\hline
\end{tabular}

(1) Referem-se às avaliações visuais de controle expressas em dias após as aplicações dos tratamentos herbicidas.

(2) Refere-se à dose de produto herbicida apresentado comercialmente.

(3) Refere-se à concentração de produto herbicida comercial na calda (v/v)

(4) Equação não significativa ao nível de $5 \%$ de probabilidade. 


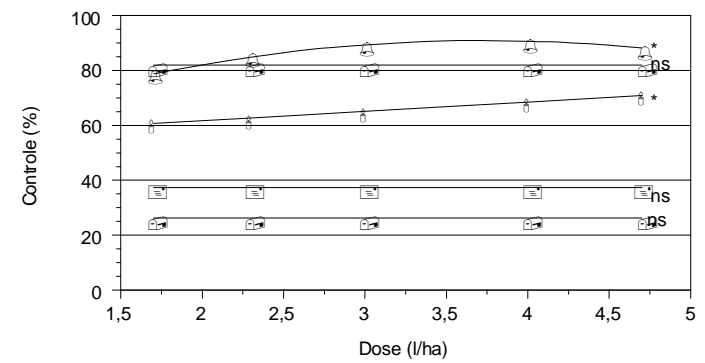

(Concentração da calda constante - $2 \%$ v/v)

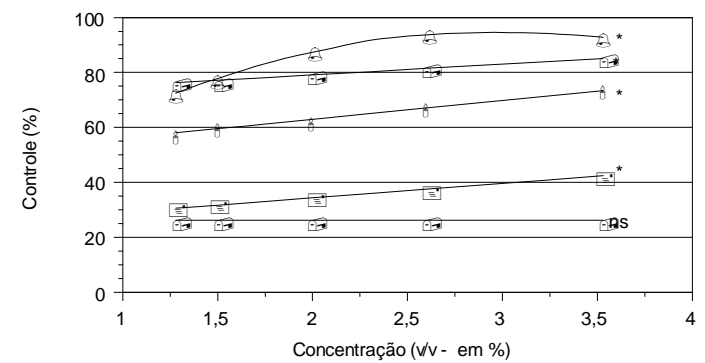

(Dose herbicida constante - 3,0 l/ha)

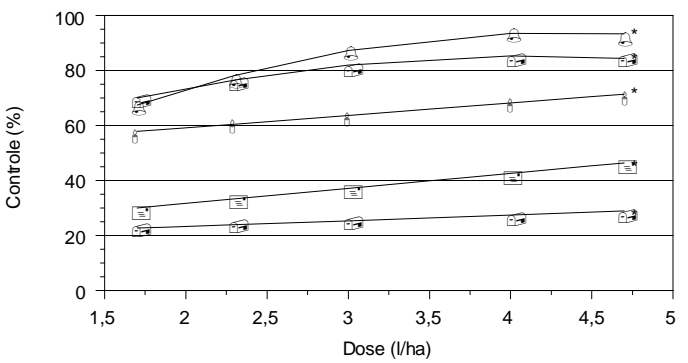

(Volume de calda constante - $150 \mathrm{l} / \mathrm{ha}$ )

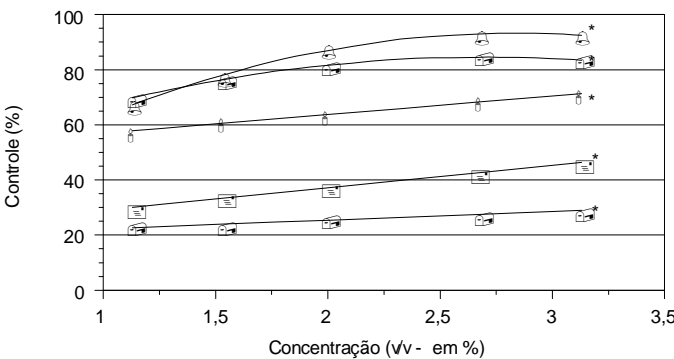

(Volume da calda constante - $150 \mathrm{l} / \mathrm{ha}$ )

FIGURA 1. Efeitos de parâmetros de aplicação na ação dessecante do herbicida sulfosate sobre arroz daninho (Oryza sativa L.), IRGA, Cachoeirinha/RS, 1994/95.

Avaliações visuais de controle realizadas aos 5, 10, 15, 20 e 25 dias após as aplicações herbicidas; em cada gráfico, a curva inferior corresponde à primeira avaliação e a superior à última delas.

(* = regressão significativa a 5\% de probabilidade; ns = regressão não significativa).

\section{LITERATURA CITADA}

AL-KHATIB, K.; GEALY, D. R.; BOERBOOM, C. M. Effect of thifensulfuron concentration and droplet size on phytotoxicity, absorption, and translocation in pea (Pisum sativum). Weed Sci., v.42, n.3, p.482-486, 1994.

AMBACH, R. M.; ASHFORD, R. Effects of variations in drop makeup on the phytotoxicity of glyphosate. Weed Sci., v.30, n.3, p.221-224, 1982.

BRYSON, C.T.; HANKS, J.E.; WILLS, G.D. Purple nutsedge (Cyperus rotundus) control in reduced-tillage cotton (Gossypium hirsutum L.) with low-volume technology.
Weed Technol., v.8, n.1, p.28-31, 1994.

BUHLER, D.D.; BURNSIDE, O.C. Effect of spray components on glyphosate toxicity to annual grasses. Weed Sci., v.31, n.1, p.124-130, 1983.

BUHLER, D. D.; BURNSIDE, O. C. Effect of application factors on post emergence phytotoxicity of fluazifop-butyl, haloxyfopmetyl, and sethoxydin. Weed Sci., v.32, n.5, p.574-583, 1984.

BUHLER, D.D.; BURNSIDE, O. C. Effect of application variables on glyphosate 
phytotoxicity. Weed Technol., v.1, n.1, p.14-17, 1987.

CARLSON, K.L.; BURNSIDE, O.C. Comparative phytotoxicity of glyphosate, SC-0224, SC0545, and HOE-00661. Weed Sci., v.32, n.6, p.841-844, 1984.

FOLONI, L. L. Avaliação de sulfosate no controle de arroz vermelho, em aplicação de pré-plantio, com diferentes doses e volumes, no sistema de plantio direto de arroz. In: ZAPP: O desafio do novo-São Paulo, Zeneca Agrícola, 1996, p.81-92.

JORDAN, T. N. Effect of diluent volumes and surfactant on the phytotoxicity of glyphosate to bermudagrass (Cynodon dactylon). Weed Sci., v.29, n.1, p. 79-83, 1981.

LEIF III, J.W.; OELKE, E.A. Effects of glyphosate and surfactant concentrations on giant burseed (Sparganium eurycarpum) control with a ropewick applicator. Weed Technol., v.4, n.3, p.625-630, 1990.
MAROCHI, A. I. Influência do horário de aplicação e volume no controle de Avena sativa (Aveia branca) em condições de seca com herbicidas sulfosate e glyphosate no sistema de plantio direto na palha. In: ZAPP: O desafio do novo, Zeneca Agrícola, São Paulo, 1996, p.67-80.

McWHORTER C. G.; GEBHARDT, M. R. Methods of applying herbicides. Weed Science Society of America, Champaign, n.4, 1987, 358p.

PRASAD, R.; CADOGAN, B. L. Influence of droplet size and density on phytotoxicity of three herbicides. Weed Technol, v.6, n.2, p.415-423, 1992.

SANDBERG, C.L.; MEGGITT, W.F.; PENNER, D. Effect of diluent volume and calcium on glyphosate phytotoxicity. Weed Sci., v.26, n.5, p.476-479, 1978.

SPRAYING SYSTEMS CO. TEEJET, Produtos de pulverização para agricultura, Spraying systems Co., Cat. 44M-P, 80 p., 1994. 